\title{
Complex-order dynamics in hexapod locomotion
}

\author{
Manuel F. Silva*, J.A. Tenreiro Machado, Ramiro S. Barbosa \\ Department of Electrical Engineering, Institute of Engineering of Porto, Rua Dr. António Bernardino de Almeida, 4200-072 Porto, Portugal
}

Received 1 June 2005; received in revised form 11 October 2005; accepted 9 December 2005

Available online 10 March 2006

\begin{abstract}
This paper studies the dynamics of foot-ground interaction in hexapod locomotion systems. For that objective the robot motion is characterized in terms of several locomotion variables and the ground is modelled through a non-linear springdashpot system, with parameters based on the studies of soil mechanics. Moreover, it is adopted an algorithm with footforce feedback to control the robot locomotion. A set of model-based experiments reveals the influence of the locomotion velocity on the foot-ground transfer function, which presents complex-order dynamics.

(C) 2006 Elsevier B.V. All rights reserved.
\end{abstract}

Keywords: Hexapod robot; Fractional-order dynamics; Fractional calculus; Locomotion; Modelling; Simulation; PD control

\section{Introduction}

Walking machines allow locomotion in terrain inaccessible to other type of vehicles, since they do not need a continuous support surface. On the other hand, the requirements for leg coordination and control impose difficulties beyond those encountered in wheeled robots [1]. There exists a class of walking machines for which walking is a natural dynamic mode. Once started on a shallow slope, a machine of this class will settle into a steady gait, without active control or energy input [2,3]. the capabilities of these machines are quite limited. Previous studies focused mainly in the control at the leg level and leg coordination using neural networks [4], fuzzy logic [4,5], hybrid force/position control [6] and subsumption architecture [7,8]. There is also a growing interest in using insect locomotion

\footnotetext{
*Corresponding author.

E-mail addresses: mss@isep.ipp.pt (M.F. Silva), jtm@isep.ipp.pt (J.A.T. Machado), rsb@isep.ipp.pt (R.S. Barbosa).
}

schemes to control walking robots at the leg level and leg coordination [9-14]. Nevertheless, the control at the joint level is almost always implemented using a PD or a PID scheme. The application of the theory of fractional calculus in robotics joint control is still in a research stage, but the recent progress in this area reveals promising aspects for future developments [15-17].

In this line of thought, it was developed a simulation model for multi-leg locomotion systems and several periodic gaits $[1,18,19]$. Based on this tool, the present article evaluates foot-ground interaction during the robot locomotion, for several walking conditions, and analyzes its dynamics in the viewpoint of fractional calculus. The main interest of this study stems from previous works showing that fractional dynamics arise in systems with "mixed" characteristics, such as the cases of a liquid interaction with a porous wall [20], in biological systems where there is the growth of tumors in healthy tissues [21] and in backlash systems with continuous-discrete interactions [22]. 
The system under analysis reveals a behavior of this kind, namely with multiple periodic collisions among the robot feet and the ground. For example, at the beginning of the support phase of each foot, although not desirable, often the contact of the foot with the ground is established and lost several times before stabilizing.

Bearing these facts in mind, the paper is organized as follows: Section 2 introduces the hexapod kinematic model and the motion planning scheme. Sections 3 and 4 present the robot dynamic model and control architecture and the locomotion system transfer function, respectively. Section 5 develops a set of experiments that reveal the system complex-order transfer functions, during locomotion at different robot forward velocities. Finally, Section 6 outlines the main conclusions and directions towards future developments.

\section{Kinematics and trajectory planning}

We consider a hexapod walking system (Fig. 1) with $n=6$ legs, equally distributed along both sides of the robot body, having each one two rotational joints (i.e., $j=\{1,2\} \equiv\{$ hip, knee $\}$ ) [19].

Motion is described by means of a world coordinate system. The kinematic model comprises: the cycle time $T$, the duty factor $\beta$, the transference time $t_{\mathrm{T}}=(1-\beta) T$, the support time $t_{\mathrm{S}}=\beta T$, the step length $L_{\mathrm{S}}$, the stroke pitch $S_{\mathrm{P}}$, the body height $H_{\mathrm{B}}$, the maximum foot clearance $F_{\mathrm{C}}$, the $i$ th leg lengths $L_{i 1}$ and $L_{i 2}$ and the foot trajectory offset $O_{i}(i=1, \ldots, n)$. Moreover, we consider a periodic trajectory for each foot, with body velocity $V_{\mathrm{F}}=L_{\mathrm{S}} / T$.

Gaits describe sequences of leg movements, alternating between transfer and support phases. Given the particular gait and the duty factor $\beta$, it is possible to calculate, for leg $i$, the corresponding phase $\phi_{i}$, the time instant where each leg leaves and returns to contact with the ground and the Cartesian trajectories of the tip of the feet (that must be completed during $t_{\mathrm{T}}$ ) [1]. Based on this data, the trajectory generator is responsible for producing a motion that synchronises and coordinates the legs.

The robot body, and by consequence the legs hips, is assumed to have a desired horizontal movement with a constant forward speed $V_{\mathrm{F}}$. Therefore, for leg $i$, the Cartesian coordinates of the hip of the legs are given by $\mathbf{p}_{\mathrm{Hd}}(t)=\left[x_{i \mathrm{Hd}}(t)\right.$, $\left.y_{i \mathrm{Hd}}(t)\right]^{\mathrm{T}}$ :

$\mathbf{p}_{\mathrm{Hd}}(t)=\left[\begin{array}{ll}V_{\mathrm{F}} t & H_{\mathrm{B}}\end{array}\right]^{\mathrm{T}}$.

Regarding the feet trajectories, for each cycle, the desired trajectory of the foot of the swing leg is computed through a cycloid function (2). For example, considering that the transfer phase starts

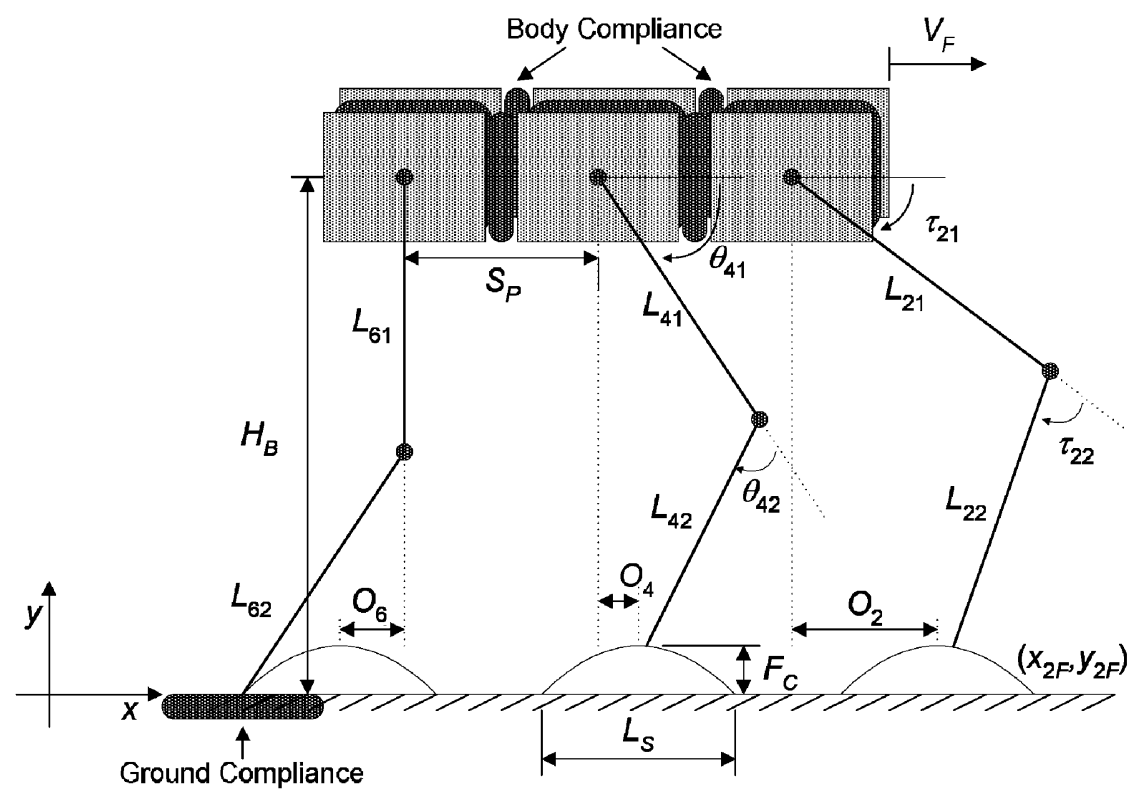

Fig. 1. Kinematic and dynamic hexapod robot model. 
at $t=0 \mathrm{~s}$, for leg $i=1$ it yields $\mathbf{p}_{\mathrm{Fd}}(t)=$ $\left[x_{i \mathrm{Fd}}(t), y_{i \mathrm{Fd}}(t)\right]^{\mathrm{T}}$ :

- During the transfer phase

$$
\begin{aligned}
\mathbf{p}_{\mathrm{Fd}}(t)= & {\left[V_{\mathrm{F}}\left[t-\frac{T}{2 \pi} \sin \left(\frac{2 \pi t}{T}\right)\right],\right.} \\
& \left.\frac{F_{\mathrm{C}}}{2}\left[1-\cos \left(\frac{2 \pi t}{T}\right)\right]\right]^{\mathrm{T}} .
\end{aligned}
$$

- During the stance phase

$$
\mathbf{p}_{\mathrm{Fd}}(t)=\left[\begin{array}{lll}
V_{\mathrm{F}} & 0
\end{array}\right]^{\mathrm{T}} \text {. }
$$

The algorithm for the forward motion planning accepts, as inputs, the desired Cartesian trajectories of the leg hips $\mathbf{p}_{\mathrm{Hd}}(t)$ and feet $\mathbf{p}_{\mathrm{Fd}}(t)$ and, by means of an inverse kinematics algorithm $\psi^{-1}$, generates as outputs the joint trajectories $\boldsymbol{\Theta}_{\mathrm{d}}(t)=$ $\left[\theta_{i 1 \mathrm{~d}}(t), \theta_{i 2 \mathrm{~d}}(t)\right]^{\mathrm{T}}$ :

$\mathbf{p}_{\mathrm{d}}(t)=\left[\begin{array}{ll}x_{i \mathrm{~d}}(t) & y_{i \mathrm{~d}}(t)\end{array}\right]^{\mathrm{T}}=\mathbf{p}_{\mathrm{Hd}}(t)-\mathbf{p}_{\mathrm{Fd}}(t)$,

$\mathbf{p}_{\mathrm{d}}(t)=\psi\left[\boldsymbol{\Theta}_{\mathrm{d}}(t)\right] \Rightarrow \boldsymbol{\Theta}_{\mathrm{d}}(t)=\psi^{-1}\left[\mathbf{p}_{\mathrm{d}}(t)\right]$,

$\dot{\boldsymbol{\Theta}}_{\mathrm{d}}(t)=\mathbf{J}^{-1}\left[\dot{\mathbf{p}}_{\mathrm{d}}(t)\right], \quad \mathbf{J}=\frac{\partial \psi}{\partial \boldsymbol{\Theta}}$.

In this study it is adopted the mammal leg configuration, namely selecting in $\psi^{-1}$ the solution corresponding to a forward knee.

In order to avoid the impact and friction effects, at the planning phase null velocities of the feet are considered in the instants of landing and taking off, assuring also the velocity continuity.

\section{Dynamics and control architecture}

\subsection{Inverse dynamics computation}

The planned joint trajectories constitute the reference for the robot control system. The model for the robot inverse dynamics is formulated as

$\boldsymbol{\Gamma}=\mathbf{H}(\boldsymbol{\Theta}) \ddot{\Theta}+\mathbf{c}(\boldsymbol{\Theta}, \dot{\boldsymbol{\Theta}})+\mathbf{g}(\boldsymbol{\Theta})-\mathbf{F}_{\mathrm{RH}}-\mathbf{J}^{\mathrm{T}}(\boldsymbol{\Theta}) \mathbf{F}_{\mathrm{RF}}$,

where $\boldsymbol{\Gamma}=\left[f_{i x}, f_{i y}, \tau_{i 1}, \tau_{i 2}\right]^{\mathrm{T}}(i=1, \ldots, n)$ is the vector of forces/torques, $\boldsymbol{\Theta}=\left[x_{i \mathrm{H}}, y_{i \mathrm{H}}, \theta_{i 1}, \theta_{i 2}\right]^{\mathrm{T}}$ is the vector of position coordinates, $\mathbf{H}(\boldsymbol{\Theta})$ is the inertia matrix and $\mathbf{c}(\boldsymbol{\Theta}, \dot{\boldsymbol{\Theta}})$ and $\mathbf{g}(\boldsymbol{\Theta})$ are the vectors of centrifugal/Coriolis and gravitational forces/torques, respectively. The $(m+2) \times 2$ (in our case $m=2$ ) matrix $\mathbf{J}^{\mathrm{T}}(\boldsymbol{\Theta})$ is the transpose of the robot
Jacobian matrix, $\mathbf{F}_{\mathrm{RH}}$ is the $(m+2) \times 1$ vector of the body inter-segment forces and $\mathbf{F}_{\mathrm{RF}}$ is the $2 \times 1$ vector of the reaction forces that the ground exerts on the robot feet. These forces are null during the foot transfer phase.

We consider that the joint actuators are not ideal, exhibiting a saturation given by

$\tau_{i j \mathrm{~m}}= \begin{cases}\tau_{i j \mathrm{C}}, & \left|\tau_{i j \mathrm{~m}}\right| \leqslant \tau_{i j \mathrm{Max}}, \\ \operatorname{sgn}\left(\tau_{i j \mathrm{C}}\right) \tau_{i j \mathrm{Max}}, & \left|\tau_{i j \mathrm{~m}}\right|>\tau_{i j \mathrm{Max}},\end{cases}$

where for leg $i$ and joint $j, \tau_{i j \mathrm{C}}$ is the controller demanded torque, $\tau_{i j \mathrm{Max}}$ is the maximum torque that the actuator can supply and $\tau_{i j \mathrm{~m}}$ is the motor effective torque.

\subsection{Robot body model}

Fig. 1 presents the dynamic model for the hexapod body and foot-ground interaction. It is considered a compliant robot body because most vertebrate walking animals have a spine that allows supporting the locomotion with improved stability.

The robot body is divided in $n$ identical segments (each with mass $M_{\mathrm{b}} n^{-1}$ ) and a linear spring-damper system is adopted to implement the intra-body compliance according to

$f_{i x \mathrm{H}}=\sum_{i^{\prime}=1}^{u}\left[-K_{x \mathrm{H}}\left(x_{i \mathrm{H}}-x_{i^{\prime} \mathrm{H}}\right)-B_{x \mathrm{H}}\left(\dot{x}_{i \mathrm{H}}-\dot{x}_{i^{\prime} \mathrm{H}}\right)\right]$,

$f_{i y \mathrm{H}}=\sum_{i^{\prime}=1}^{u}\left[-K_{y \mathrm{H}}\left(y_{i \mathrm{H}}-y_{i^{\prime} \mathrm{H}}\right)-B_{y \mathrm{H}}\left(\dot{y}_{i \mathrm{H}}-\dot{y}_{i^{\prime} \mathrm{H}}\right)\right]$,

where $\left(x_{i^{\prime} \mathrm{H}}, y_{i^{\prime} \mathrm{H}}\right)$ are the hip coordinates and $u$ is the total number of segments adjacent to leg $i$, respectively. The parameters $K_{\eta \mathrm{H}}$ and $B_{\eta \mathrm{H}}$ $(\eta=\{x, y\}$ in the $\{$ horizontal, vertical $\}$ directions, respectively) are defined so that the body behavior is similar to the one expected to occur on an animal (Table 1).

\subsection{Foot-ground interaction model}

The contact of the $i$ th robot foot with the ground is modelled (see Fig. 1) through a non-linear system [23] with linear stiffness $K_{\eta \mathrm{F}}$ and non-linear damping $B_{\eta \mathrm{F}}(\eta=\{x, y\}$ in the \{horizontal, vertical\} 
directions, respectively) yielding:

$$
\begin{aligned}
f_{i x \mathrm{~F}}= & -K_{x \mathrm{~F}}\left(x_{i \mathrm{~F}}-x_{i \mathrm{~F} 0}\right) \\
& -B_{x \mathrm{~F}}\left[-\left(y_{i \mathrm{~F}}-y_{i \mathrm{~F} 0}\right)\right]\left(\dot{x}_{i \mathrm{~F}}-\dot{x}_{i \mathrm{~F} 0}\right), \\
f_{i y \mathrm{~F}}= & -K_{y \mathrm{~F}}\left(y_{i \mathrm{~F}}-y_{i \mathrm{~F} 0}\right) \\
& -B_{y \mathrm{~F}}\left[-\left(y_{i \mathrm{~F}}-y_{i \mathrm{~F} 0}\right)\right]^{v}\left(\dot{y}_{i \mathrm{~F}}-\dot{y}_{i \mathrm{~F} 0}\right),
\end{aligned}
$$

where $x_{i \mathrm{~F} 0}$ and $y_{i \mathrm{~F} 0}$ are the coordinates of foot $i$ touchdown and the exponent $v$ of the non-linear dashpot is a parameter dependent on the ground characteristics. The values for the parameters $K_{\eta \mathrm{F}}$ and $B_{\eta \mathrm{F}}$ (Table 1) are based on the studies of soil mechanics [23].

\subsection{Control architecture}

The general control architecture of the multilegged locomotion system is presented in Fig. 2. The trajectory planning is held in the Cartesian space, but the control is performed in the joint space,

Table 1

System parameters

\begin{tabular}{llll}
\hline Robot model parameters & \multicolumn{2}{l}{ Locomotion parameters } \\
\hline$S_{\mathrm{P}}(\mathrm{m})$ & 1.0 & $\beta(\%)$ & 50 \\
$L_{\mathrm{ij}}(\mathrm{m})$ & 0.5 & $L_{\mathrm{S}}(\mathrm{m})$ & 1.0 \\
$O_{i}(\mathrm{~m})$ & 0.0 & $H_{\mathrm{B}}(\mathrm{m})$ & 0.9 \\
$M_{\mathrm{b}}(\mathrm{kg})$ & 88.0 & $F_{\mathrm{C}}(\mathrm{m})$ & 0.1 \\
$M_{i j}(\mathrm{~kg})$ & 1.0 & $V_{\mathrm{F}}\left(\mathrm{m} \mathrm{s}^{-1}\right)$ & 1.0 \\
$M_{i \mathrm{f}}\left(\mathrm{kg}^{-1}\right)$ & 0.0 & Ground parameters \\
$k_{x \mathrm{H}}\left(\mathrm{N} \mathrm{m}^{-1}\right)$ & $10^{5}$ & $k_{x \mathrm{~F}}\left(\mathrm{~N} \mathrm{~m}^{-1}\right)$ & 1302152.0 \\
$k_{y \mathrm{H}}\left(\mathrm{N} \mathrm{m}^{-1}\right)$ & $10^{4}$ & $k_{y \mathrm{~F}}\left(\mathrm{~N} \mathrm{~m}^{-1}\right)$ & 1705199.0 \\
$B_{x \mathrm{H}}\left(\mathrm{N} \mathrm{s} \mathrm{m}^{-1}\right)$ & $10^{3}$ & $B_{x \mathrm{~F}}\left(\mathrm{~N} \mathrm{~s} \mathrm{~m}^{-1}\right)$ & 2364932.0 \\
$B_{y \mathrm{H}}\left(\mathrm{N} \mathrm{s} \mathrm{m}^{-1}\right)$ & $10^{2}$ & $B_{y \mathrm{~F}}\left(\mathrm{~N} \mathrm{~s} \mathrm{~m}^{-1}\right)$ & 2706233.0 \\
& & $v$ & 0.9 \\
\hline
\end{tabular}

which requires the integration of the inverse kinematic model in the forward path. The control algorithm considers an external position and velocity feedback and an internal feedback loop with information of foot-ground interaction force.

On a previous work it was demonstrated the superior performance of introducing force feedback and this was highlighted for the case of having nonideal actuators with saturation or variable ground characteristics [23].

Based on these results, in this study we adopt a PD controller for $G_{\mathrm{cl}}(s)$ and a simple P controller for $G_{\mathrm{c} 2}$. For the PD algorithm we have

$G_{\mathrm{C} 1 j}(s)=K_{\mathrm{p}_{j}}+K_{\mathrm{d}_{j}} s, \quad j=1,2$

being $K_{\mathrm{p}_{j}}$ and $K_{\mathrm{d}_{j}}$ the proportional and derivative gains, respectively.

\section{Locomotion system transfer function}

In order to obtain the transfer functions (TF) of the system (i.e., the robot and the environment), the frequency response of the locomotion system is computed numerically. For that purpose, small amplitude sinusoidal exciting signals $\delta \mathbf{p}_{\mathrm{d}}(t)$ are superimposed, separately, on the frequency range under analysis, over the $x$ and $y$ feet desired Cartesian trajectories, according to the block diagram presented in Fig. 3. The resulting feet reference trajectories are given by

$$
\begin{aligned}
\mathbf{p}_{\mathrm{d}}(t)+\delta \mathbf{p}_{\mathrm{d}}(t) & =\left[\begin{array}{c}
x_{i \mathrm{~d}}(t)+\delta x_{i \mathrm{~d}}(t) \\
y_{i \mathrm{~d}}(t)+\delta y_{i \mathrm{~d}}(t)
\end{array}\right], \\
\mathbf{p}_{\mathrm{d}}(t)+\boldsymbol{\delta} \mathbf{p}_{\mathrm{d}}(t) & =\psi\left[\boldsymbol{\Theta}_{\mathrm{d}}(t)+\boldsymbol{\delta} \boldsymbol{\Theta}_{\mathrm{d}}(t)\right] \Rightarrow \boldsymbol{\Theta}_{\mathrm{d}}(t)+\boldsymbol{\delta} \boldsymbol{\Theta}_{\mathrm{d}}(t) \\
& =\boldsymbol{\psi}^{-1}\left[\mathbf{p}_{\mathrm{d}}(t)+\boldsymbol{\delta} \mathbf{p}_{\mathrm{d}}(t)\right],
\end{aligned}
$$

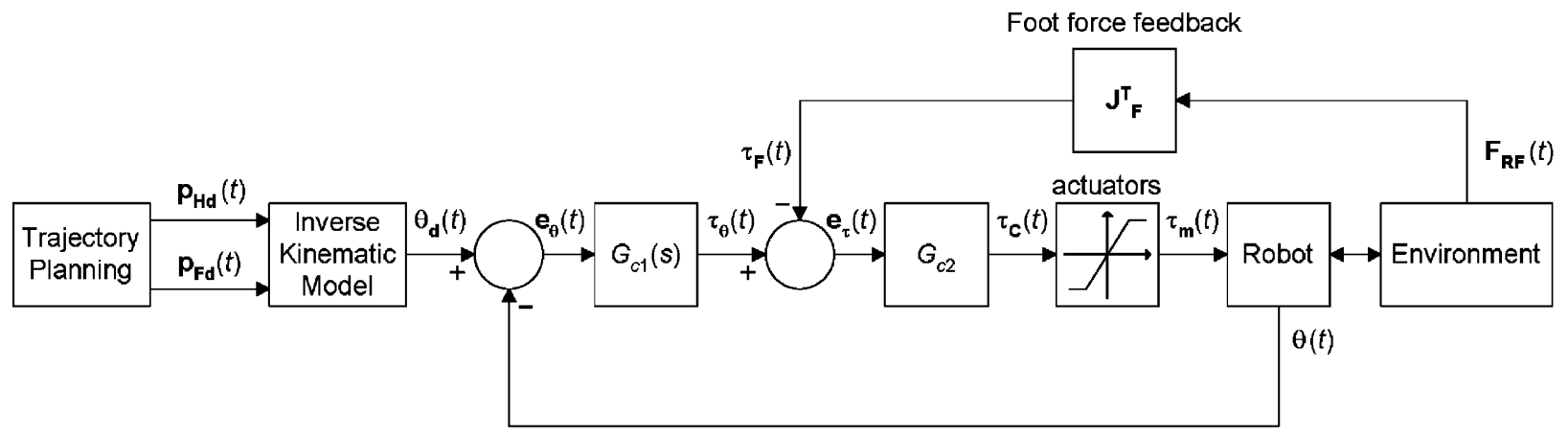

Position / velocity feedback

Fig. 2. Hexapod robot control architecture. 


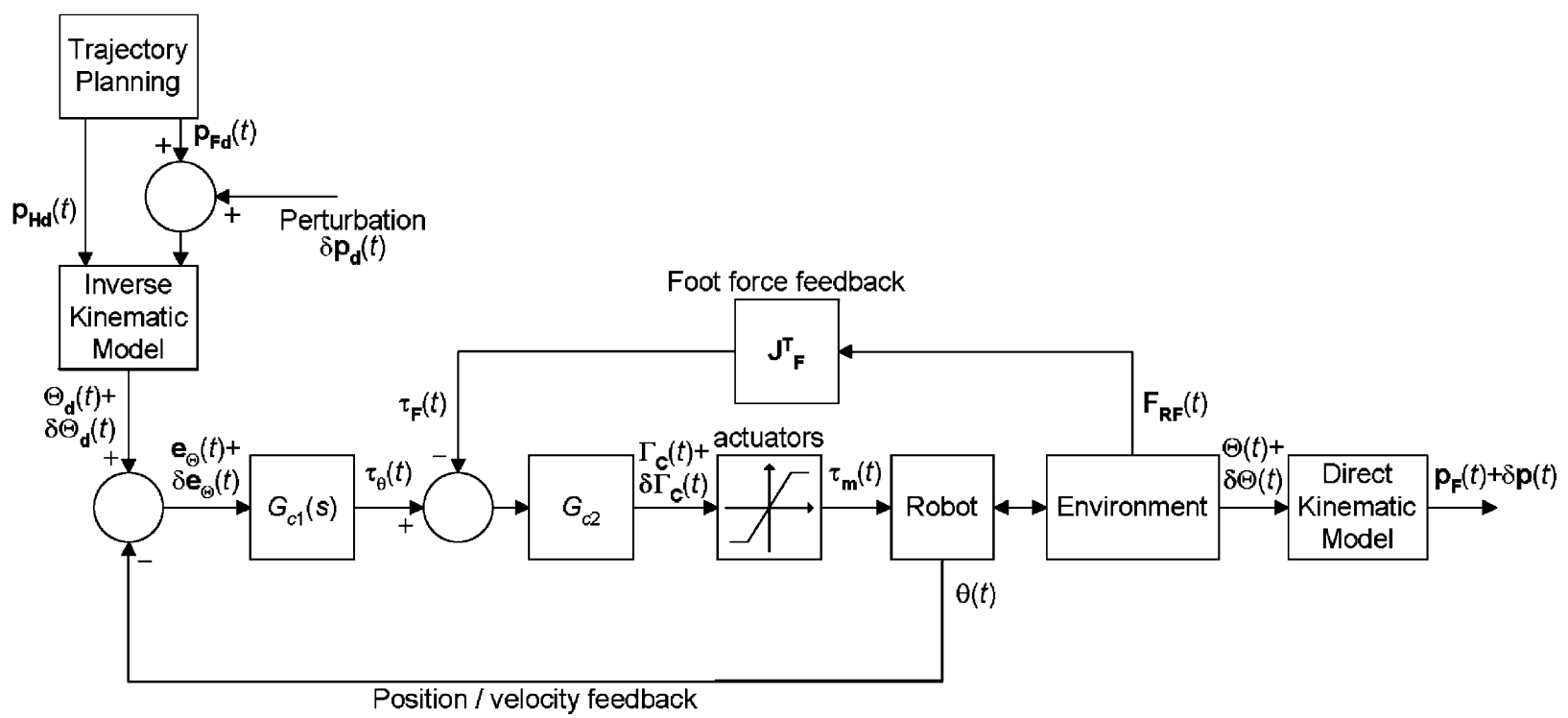

Fig. 3. Block diagram adopted for the calculation of the transfer functions $G_{x j}(s)$ and $G_{y j}(s), j=1,2$.

where $\mathbf{p}_{\mathrm{d}}(t)+\boldsymbol{\delta} \mathbf{p}_{\mathrm{d}}(t)$ are the $i$ th feet desired Cartesian trajectories (relatively to their hip) perturbed with a sinusoidal signal of small amplitude and $\boldsymbol{\Theta}_{\mathrm{d}}(t)+\boldsymbol{\delta} \boldsymbol{\Theta}_{\mathrm{d}}(t)$, are the corresponding perturbed joint trajectories. During the robot locomotion simulation, the perturbations propagate to the torques demanded to the robot leg joint actuators by the controller (resulting $\boldsymbol{\Gamma}_{\mathrm{C}}(t)+\boldsymbol{\delta} \boldsymbol{\Gamma}_{\mathrm{C}}(t)$ ) and to the robot real feet trajectories (that become $\left.\mathbf{p}_{\mathrm{F}}(t)+\boldsymbol{\delta} \mathbf{p}(t)\right)$.

The system TFs are given by $(j=1,2)$

$G_{x j}(\mathrm{j} \omega)=\frac{F\left\{\delta x_{1 \mathrm{~F}}(t)\right\}}{F\left\{\delta \tau_{1 j \mathrm{C}}(t)\right\}}$,

$G_{y j}(\mathrm{j} \omega)=\frac{F\left\{\delta y_{1 \mathrm{~F}}(t)\right\}}{F\left\{\delta \tau_{1 j \mathrm{C}}(t)\right\}}$,

where $\delta x_{1 \mathrm{~F}}(t)$ and $\delta y_{1 \mathrm{~F}}(t)$ are the resulting leg 1 foot trajectory perturbations, $\delta \tau_{11 \mathrm{C}}(t)$ and $\delta \tau_{12 \mathrm{C}}(t)$ are the corresponding joint demanded torques perturbations and $F\{\}$ represents the Fourier Transform operator.

\section{Simulation results}

In this section, we develop a set of simulations to analyze the TFs of the hexapod-environment system for two different velocities, namely $V_{\mathrm{F}}=1.0 \mathrm{~m} \mathrm{~s}^{-1}$ and $V_{\mathrm{F}}=2.0 \mathrm{~m} \mathrm{~s}^{-1}$. We consider the robot body parameters, the locomotion parameters and the ground parameters presented in Table 1 and joint actuators with a maximum torque in (8) of $\tau_{i j \operatorname{Max}}=400 \mathrm{Nm}$.

In all simulations the discrete-time control algorithm is evaluated with a sampling frequency of $f_{\text {sc }}=2.0 \mathrm{kHz}$ while the robot and environment dynamics are calculated with a sampling frequency of $f_{\mathrm{sr}}=20.0 \mathrm{kHz}$.

\subsection{Controller tuning methodology}

To tune the controller we adopt a systematic method, testing and evaluating a grid of several possible combinations of controller parameters, while establishing a compromise in what concerns the simultaneous minimization of the mean absolute energy per travelled distance and the hips trajectory following errors [23].

The resulting controller parameters are presented in Table 2, for a proportional controller $G_{\mathrm{c} 2}$ with gain $K \mathrm{p}_{j}=0.9$.

\subsection{Transfer function computation}

In order to determine $G_{x j}$ and $G_{y j}(j=1,2)$, the TFs of the robot-environment, the locomotion is simulated while the robot is moving on a perfectly flat surface without obstacles in its path. For this purpose, sinusoidal perturbations, with maximum amplitudes of $\delta x_{i \mathrm{~d}}(t)=10^{-4} \mathrm{~m}$ and $\delta y_{i \mathrm{~d}}(t)=10^{-4} \mathrm{~m}$ in the $x$ and $y$ directions, respectively, are superimposed, separately, over the planned robot feet 
Cartesian trajectories, in the range of frequencies $0.001 \mathrm{rad} \mathrm{s}^{-1} \leqslant \omega \leqslant 100.0 \mathrm{rad} \mathrm{s}^{-1}$ during $T_{\text {sim }} \approx 40000$ steps. Fig. 4 presents charts of the sinusoidal perturbation $\delta x_{i \mathrm{~d}}(t)$, for $\omega=100.0 \mathrm{rad} \mathrm{s}^{-1}$, and the corresponding feet trajectory perturbations $\delta x_{1 \mathrm{~F}}(t)$ and joint torque perturbations $\delta \tau_{11 \mathrm{C}}(t)$ and $\delta \tau_{12 \mathrm{C}}(t)$.

We start with $G_{x j}$ for a robot forward locomotion speed of $V_{\mathrm{F}}=1.0 \mathrm{~m} \mathrm{~s}^{-1}$. As can be observed from the Nichols chart presented in Fig. 5, $G_{x 1}$ presents different asymptotes for different frequency ranges.

Table 2

$G_{\mathrm{cl}}(s)$ controller parameters

\section{PD}

\begin{tabular}{llr}
\hline Joint $j=1$ & $K \mathrm{p}_{1}$ & 4500 \\
& $K \mathrm{~d}_{1}$ & 110 \\
Joint $j=2$ & $K \mathrm{p}_{2}$ & 1500 \\
& $K \mathrm{~d}_{2}$ & 20 \\
\hline
\end{tabular}
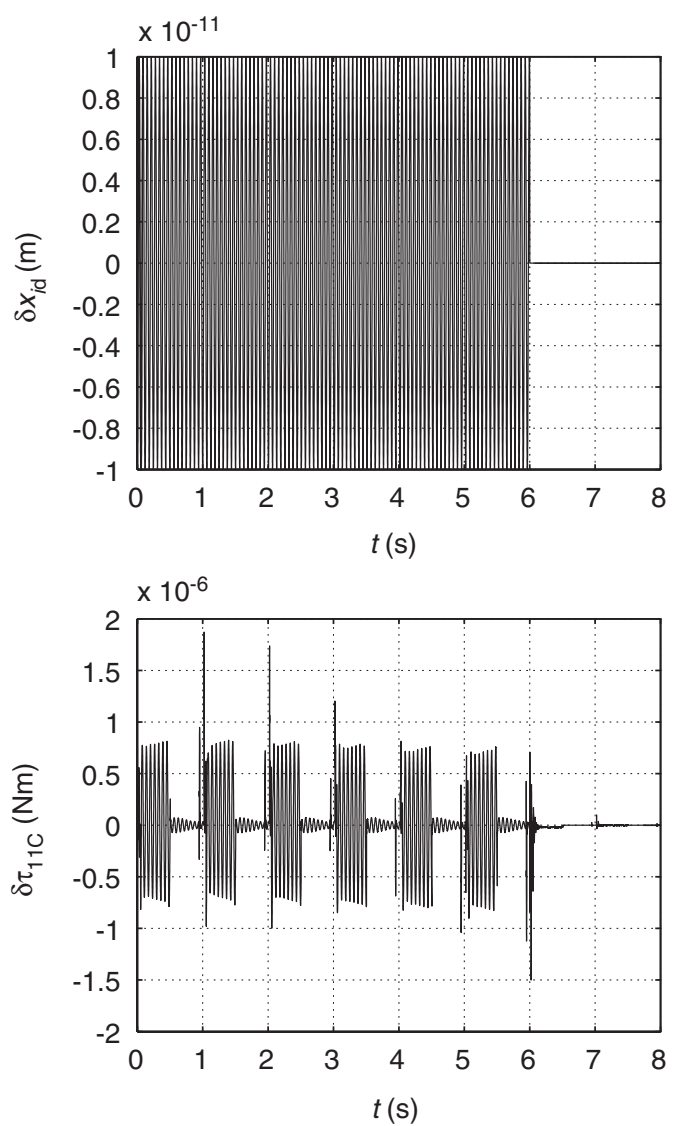

At low frequencies $\left(A \equiv[0.001 ; 0.05] \mathrm{rad} \mathrm{s}^{-1}\right)$ the asymptote can be approximated by

$$
\begin{aligned}
& G_{x j}(s) \approx \frac{k_{x j}}{J\left(s^{\alpha_{x j}+\jmath \beta_{x j}}\right)}, \quad J=\sqrt{-1}, \quad \alpha_{x j}, \beta_{x j} \in \mathfrak{R}, \\
& j=1,2 .
\end{aligned}
$$

The values of the parameters $\alpha_{x j}$ and $\beta_{x j}$ for the low frequency asymptotic approximation of $G_{x 1}$ are presented in Table 3.

At medium and at high frequencies (regions $\mathrm{B} \equiv$ $[0.05 ; 0.5] \mathrm{rad} \mathrm{s}^{-1}$ and $\left.\mathrm{C} \equiv[0.5 ; 5.0] \mathrm{rad} \mathrm{s}^{-1}\right)$, the resulting TFs can be approximated by an expression of the type:

$G_{x j}(s) \approx \frac{k_{x j}}{s^{\alpha_{x j}+\jmath \beta_{x j}}}, \quad \alpha_{x j}, \beta_{x j} \in \mathfrak{R}, \quad j=1,2$.

The values of the parameters $\alpha_{x j}$ and $\beta_{x j}$ for the asymptotic approximations in these frequency ranges are also presented in Table 3.
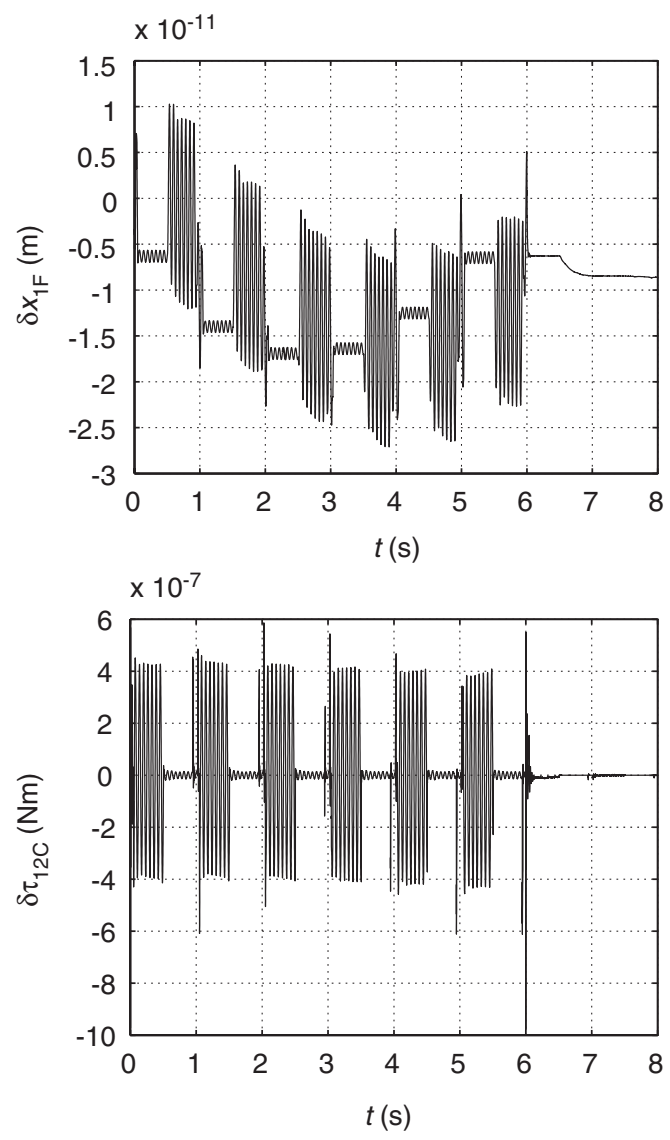

Fig. 4. Sinusoidal perturbation $\delta x_{i \mathrm{~d}}(t)$, for $\omega=100.0 \mathrm{rad} \mathrm{s}^{-1}$ (top, left), and the corresponding feet trajectory perturbations $\delta x_{1 \mathrm{~F}}(t)$ (top, right) and joint torque perturbations $\delta \tau_{11 \mathrm{C}}(t)$ (lower, left) and $\delta \tau_{12 \mathrm{C}}(t)$ (lower, right) for $V_{\mathrm{F}}=1.0 \mathrm{~m} \mathrm{~s}^{-1}$. 

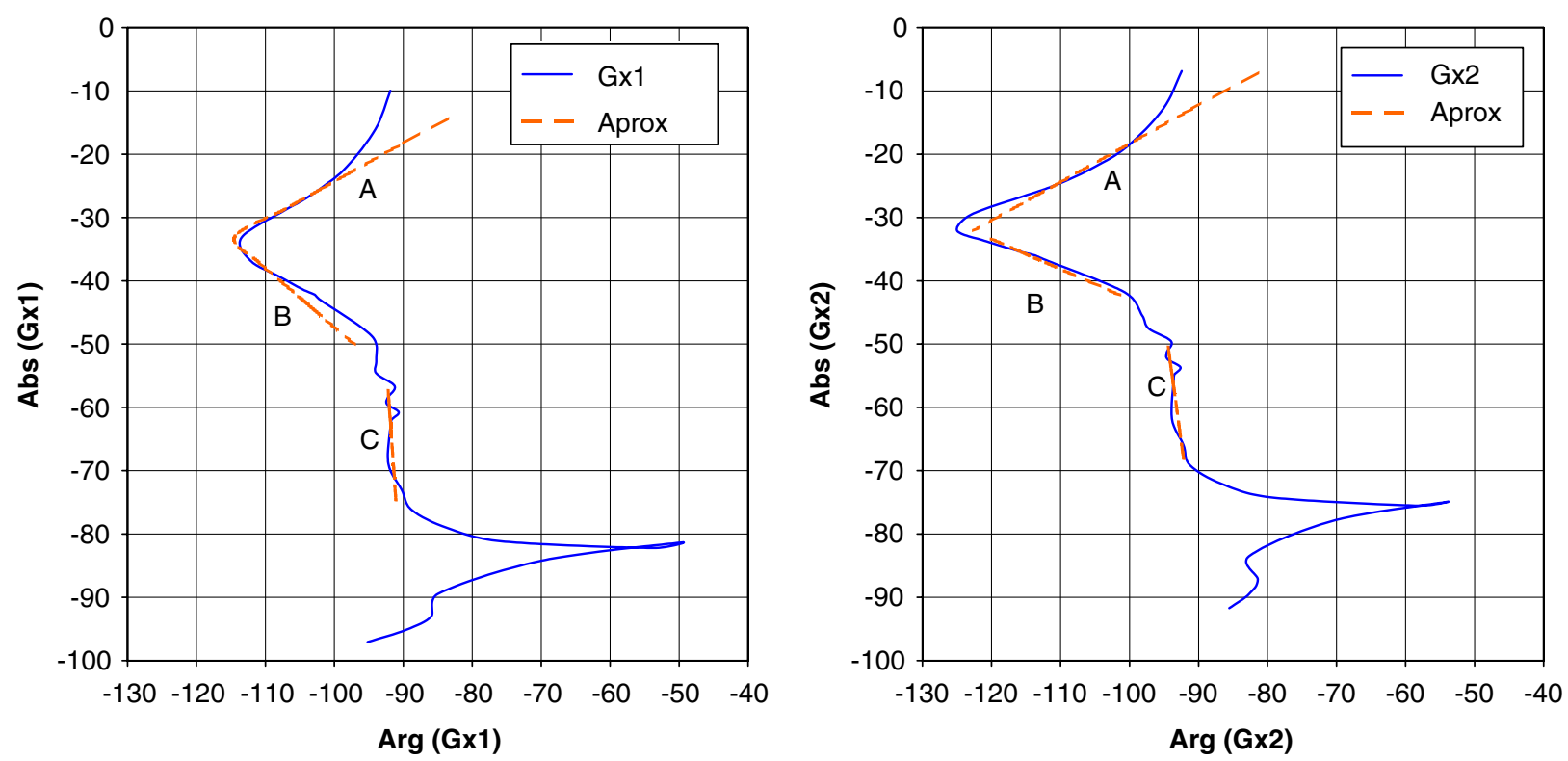

Fig. 5. Nichols charts of $G_{x 1}(\mathrm{j} \omega)$ and $G_{x 2}(\mathrm{j} \omega)$, and their approximations at low (A), medium (B) and high frequencies (C), for $V_{\mathrm{F}}=1.0 \mathrm{~m} \mathrm{~s}^{-1}$.

Table 3

Parameters values for the asymptotic approximations of the Nichols charts of $G_{x 1}$ and $G_{x 2}$, with $V_{\mathrm{F}}=1.0 \mathrm{~m} \mathrm{~s}^{-1}$

\begin{tabular}{|c|c|c|c|c|c|c|}
\hline$V_{\mathrm{F}}=1.0 \mathrm{~m} \mathrm{~s}^{-1}$ & $G_{x 1}$ & & & $G_{x 2}$ & & \\
\hline Frequency range & $k_{x 1}$ & $\alpha_{x 1}$ & $\beta_{x 1}$ & $k_{x 2}$ & $\alpha_{x 2}$ & $\beta_{x 2}$ \\
\hline Low (A) & 0.001 & 0.72 & 0.18 & 0.001 & 0.77 & 0.22 \\
\hline Medium (B) & 0.0014 & 0.84 & -0.20 & 0.0022 & 1.03 & -0.19 \\
\hline $\operatorname{High}(\mathrm{C})$ & 0.00068 & 1.02 & -0.01 & 0.002 & 1.04 & -0.04 \\
\hline
\end{tabular}

These results reveal a complex order dynamics that is a consequence of the foot-ground interaction, with several free-impact-contact-impact-free dynamical states. Complex-order dynamics have already been addressed in modelling and control [24-27].

We verify that the Nichols chart of $G_{x 2}$ has similar features to those of $G_{x 1}$, as can be observed in Fig. 5. The asymptotic approximations at low, medium and high frequencies, can be described by identical expressions and occur in the same frequency ranges as for the case of $G_{x 1}$. The same can be concluded by comparing the values of the parameters $\alpha_{x j}$ and $\beta_{x j}$, for the asymptotic approximations of $G_{x 2}$ and $G_{x 1}$ (Table 3).

In a second phase, the study is repeated for a robot velocity of $V_{\mathrm{F}}=2.0 \mathrm{~m} \mathrm{~s}^{-1}$ and the conclusions are identical. The asymptotic approximations at low, medium and high frequencies, presented in Fig. 6 for $G_{x 1}$, obey to the same expressions as in the case for $V_{\mathrm{F}}=1.0 \mathrm{~m} \mathrm{~s}^{-1}$ ((18) and (19), for low and medium and high frequencies, respectively).

However, for the locomotion velocity $V_{\mathrm{F}}=$ $2.0 \mathrm{~m} \mathrm{~s}^{-1}$ the low, medium and high frequency behaviors occur in the ranges $\mathrm{A} \equiv[0.008 ; 0.08] \mathrm{rad} \mathrm{s}^{-1}$, $\mathrm{B} \equiv[0.1 ; 0.9] \mathrm{rads}^{-1}$ and $\mathrm{C} \equiv[1.0 ; 5.0] \mathrm{rad} \mathrm{s}^{-1}$, respectively.

Moreover, we verify that the Nichols chart of $G_{x 2}$ (Fig. 6), and therefore its TF, is very similar to the one of $G_{x 1}$. The same can be concluded by comparing the values of the parameters $\alpha_{x j}$ and $\beta_{x j}$, for the asymptotic approximations of $G_{x 1}$ and $G_{x 2}$ (Table 4).

The meaning of the imaginary factor $J$ in the denominator of expression (18) in not yet clear. However, the authors believe on the existence of an 

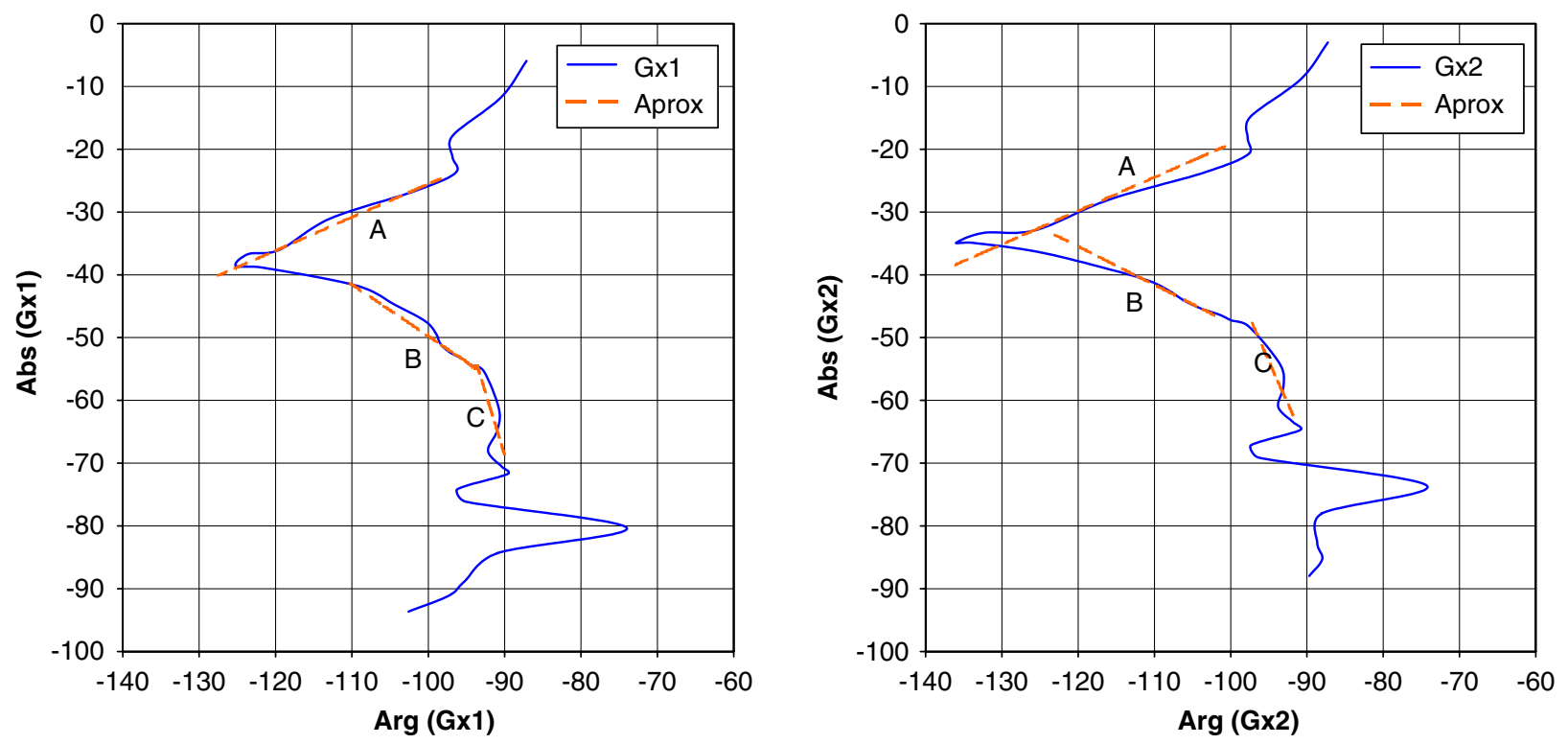

Fig. 6. Nichols charts of $G_{x 1}(\mathrm{j} \omega)$ and $G_{x 2}(\mathrm{j} \omega)$, and their approximations at low (A), medium (B) and high frequencies (C), for $V_{\mathrm{F}}=2.0 \mathrm{~m} \mathrm{~s}^{-1}$.

Table 4

Parameters values for the asymptotic approximations of the Nichols charts of $G_{x 1}$ and $G_{x 2}$, with $V_{\mathrm{F}}=2.0 \mathrm{~m} \mathrm{~s}^{-1}$

\begin{tabular}{|c|c|c|c|c|c|c|}
\hline$V_{\mathrm{F}}=2.0 \mathrm{~m} \mathrm{~s}^{-1}$ & $G_{x 1}$ & & & $G_{x 2}$ & & \\
\hline Frequency range & $k_{x 1}$ & $\alpha_{x 1}$ & $\beta_{x 1}$ & $k_{x 2}$ & $\alpha_{x 2}$ & $\beta_{x 2}$ \\
\hline Low (A) & 0.0004 & 0.96 & 0.24 & 0.0007 & 0.95 & 0.27 \\
\hline Medium (B) & 0.003 & 0.85 & -0.27 & 0.0055 & 1.09 & -0.27 \\
\hline $\operatorname{High}(\mathrm{C})$ & 0.0015 & 1.04 & 0.02 & 0.0045 & 1.08 & -0.06 \\
\hline
\end{tabular}

expression unifying the asymptotic behavior of the $\mathrm{TF}$ both at low and medium frequencies, which is currently under investigation. One possibility, under study, is to replace the $s^{\alpha+j \beta}$, that leads to complexvalued outputs, by one of the operators $H_{1}(s)=$ $s^{\alpha+\jmath \beta}+s^{\alpha-\jmath \beta}$ or $H_{2}(s)=-J\left[s^{\alpha+\jmath \beta}-s^{\alpha-\jmath \beta}\right]$ that lead to real valued outputs. Supporting this consideration we have the similarities between the values of the parameters $\alpha_{x j}$ and $\beta_{x j}$ (i.e., complex conjugate exponents for regions $A$ and $B$ ) for the low and medium frequency asymptotic approximations of $G_{x j}(j=1,2)$.

\section{Conclusions}

In this paper, we have studied the dynamics of foot-ground interaction for hexapod robots. The simulation results for different robot velocities are consistent with each other and reveal that, in the range of the locomotion velocities under consideration, this system reveals complex-order dynamics. The meaning of the complex-order dynamics and the influence of the system parameters is not yet totally clear. Moreover, questions remain on the meaning of the imaginary factor $J$ in the expression of the asymptotic approximation of the transfer function at low frequencies. In this line of thought, the authors are seeking for an unifying expression for the TFs.

While our focus has been on a dynamic analysis in periodic gaits, many aspects of locomotion are not necessarily captured by the proposed simulations. Consequently, future work will address the implementation of new experiments in order to estimate how the complex-order dynamics varies with the locomotion parameters, the ground models and the robot characteristics. 


\section{References}

[1] S.-M. Song, K. Waldron, Machines that Walk: The Adaptive Suspension Vehicle, MIT Press, Cambridge, MA, 1989.

[2] T. McGeer, Passive dynamic walking, Internat. J. Robotics Res. 9 (1990) 62-82.

[3] A.C. Smith, M.D. Berkemeier, Passive dynamic quadrupedal walking, in: Proceedings IEEE International Conference on Robotics and Automation (ICRA '97), Albuquerque, New Mexico, USA, 1997, pp. 34-39.

[4] C.-R. Tsai, T.-T. Lee, A study of fuzzy-neural force control for a quadrupedal walking machine, J. Dynam. Systems Measurement Control 120 (1998) 124-133.

[5] M.C. Birch, et al., Design of a cricket microrobot, in: Proceedings IEEE International Conference on Robotics and Automation (ICRA '00), USA, 2000, pp. 1109-1114.

[6] C.-R. Tsai, T.-T. Lee, S.-M. Song, Fuzzy logic control of a planetary gear type walking machine leg, Robotica 15 (1997) 533-546.

[7] D. Wettergreen, Robotic walking in natural terrain-gait planning and behavior-based control for statically-stable walking robots, Ph.D. Thesis, Carnegie Mellon University, December 1995.

[8] E. Celaya, J. Porta, Force-based control of a six-legged robot on abrupt terrain using the subsumption architecture, in: Proceedings of the International Conference on Advanced Robotics (ICAR '95), 1995, pp. 413-419.

[9] H.J. Chiel, R.D. Beer, R.D. Quinn, K.S. Espenschied, Robustness of a distributed neural network controller for locomotion in a hexapod robot, IEEE Trans. Robotics Automat. 8 (1992) 293-303.

[10] J.J. Collins, S.A. Richmond, Hard-wired central pattern generators for quadrupedal locomotion, Biol. Cybernet. 71 (1994) 375-385.

[11] C. Ferrell, A comparison of three insect inspired locomotion controllers, Robotics Autonomous System 16 (1995) 135-159.

[12] F. Pfeiffer, J. Eltze, H. Weidemann, The tum-walking machine (extended), Intell. Automat. Soft Comput. 1 (1995) 307-323.

[13] G. Nelson, R. Quinn, Posture control of a cockroach-like robot, IEEE Control Systems 19 (1999) 9-14.

[14] C. Zhifeng, Z. Xiuli, Z. Haojun, Z. Liyao, The cpg-based bionic quadruped system, in: Proceedings of the IEEE International Conference on Systems, Man and Cybernetics (SMC '03), Washington, USA, 2003, pp. 1828-1833.

[15] M.F. Silva, J.A.T. Machado, A.M. Lopes, Comparison of fractional and integer order control of an hexapod robot, in: Proceedings of the VIB 2003-ASME International 19th
Biennial Conference on Mechanical Vibration and Noise, ASME, Chicago, Illinois, USA, 2003.

[16] M.F. Silva, J.A.T. Machado, A.M. Lopes, Integer vs. fractional order control of a hexapod robot, in: M.A. Armada, P.G. de Santos (Eds.), Climbing and Walking Robots, CLAWAR, Springer, Madrid, Spain, 2005.

[17] M.F. Silva, J.A.T. Machado, A.M. Lopes, Fractional order control of a hexapod robot, Nonlinear Dynamics 38 (1-4) (2004) 417-433.

[18] http://www.biology.leeds.ac.uk/teaching/3rdyear/Blgy3120/ Jmvr/Loco/Gaits/GAITS.htm, 2005.

[19] M.F. Silva, J.A.T. Machado, A.M. Lopes, Modelling and simulation of artificial locomotion systems, ROBOTICA 23 (5) (2005) 595-606.

[20] A. Oustaloup, La Commande CRONE: Commande Robuste d'Ordre Non Entier, Éditions Hermès, 1991.

[21] W. Chen, S. Holm, Fractional derivative mathematical and numerical modelling of acoustic attenuations obeying arbitrary frequency power law, in: Proceedings of the Sixth International Conference on Theoretical \& Computational Acoustics, 2003.

[22] R.S. Barbosa, J.A.T. Machado, Describing function analysis of systems with impacts and backlash, Nonlinear Dynamics 29 (1-4) (2002) 235-250.

[23] M.F. Silva, J.A.T. Machado, A.M. Lopes, Position/force control of a walking robot, Mach. Intell. Robotic Control (2003) 33-44.

[24] J. Sabatier, S. Poullain, P. Latteux, J.L. Thomas, A. Oustaloup, Robust speed control of a low damped electromechanical system based on crone control: application to a four mass experimental test bench, Nonlinear Dynamics 38 (1-4) (2004) 383-400.

[25] T.T. Hartley, C.F. Lorenzo, J.L. Adams, Conjugated-order differintegrals, in: Proceedings of the VIB 2005-ASME International 20th Biennial Conference on Mechanical Vibration and Noise, ASME, Long Beach, California, USA, 2005, dECT2005-84951.

[26] R.R. Nigmatullin, J.J. Trujillo, The mesoscopic 'fractional' kinetic equations and a generalized Riemann-Liouville integral, in: D.H. van Campe, M.D. Lazurko, W.P.J.M. van den Oever (Eds.), Proceedings ENOC-2005-Fifth EUROMECH Nonlinear Dynamics Conference, EURO$\mathrm{MECH}$, Eindhoven University of Technology, Eindhoven, The Netherlands, 2005, pp. 1402-1408 (ISBN 90-386-2667-3).

[27] T.T. Hartley, J.L. Adams, C.F. Lorenzo, Complex orderdistributions, in: Proceedings of the VIB 2005-ASME International 20th Biennial Conference on Mechanical Vibration and Noise, ASME, Long Beach, California, USA, 2005 (dECT2005-84952). 